Review Article

\title{
Diabetes Mellitus and Heart Diseases
}

\author{
Afa Bayramova* \\ Department of Gynecology, Russia
}

Submission: January 23, 2018; Published: March 09, 2018

*Corresponding author: Afa Bayramova, Department of Gynecology, Russia, Tel: +79610220184; Email: afa.bayramova@yandex.ru

\begin{abstract}
Diabetes mellitus is recognized by WHO experts as a non-communicable epidemic and represents a serious medical and social problem. In 2012 , suffered of diabetes mellitus about $6.4 \%$ (285 million) of the world's inhabitants. By 2030, the number of patients is expected to increase to $7.7 \%$ (439 million people) [1]. Coronary heart disease is the leading cause of death in patients with diabetes [2], with $90 \%$ of these patients suffering from type 2 diabetes (diabetes mellitus 2) [3].
\end{abstract}

Keywords: Breast cancer; Oncogynaecology; Gynaecology; Oncology; Surgery

\section{Introduction}

The presence of diabetes is associated with the emergence of all forms of coronary heart disease - angina pectoris, painless myocardial ischemia, myocardial infarction, sudden cardiac death [4]. At the same time, macrovascular complications, including coronary heart disease, stroke, peripheral vascular disease, are the cause of death of patients with diabetes in $67 \%$ of cases [5]. In $50 \%$ of cases, the increased risk of developing cardiovascular lesions in diabetes mellitus 2 is due to the greater frequency and severity of traditional risk factors in diabetic patients [6,7].

Table 1: Prevalence of dysplicemia.
Risk factors in patients with diabetes are: dyslipidemia, arterial hypertension, smoking, hereditary predisposition for coronary heart disease, the presence of micro- and macroalbuminuria.

\section{Diabetes Mellitus and Lipid Metabolism Disorders}

A study of the prevalence, methods of diagnosis and treatment of dyslipidemia is of particular interest in patients with diabetes. Information on the prevalence of dyslipidemia in patients with diabetes 2 is presented in Table 1 [8].

\begin{tabular}{|c|c|c|c|}
\hline Indicators & Type 2 Diabetes,\% (N=359) & Without Diabetes, \% (N=1064) & P \\
\hline Triglycerides $>2.3 \mathrm{mmol} / \mathrm{l}(>200 \mathrm{mg} / \mathrm{dL})$ & 29.2 & 13.1 & $<0.001$ \\
\hline $\begin{array}{c}\text { High density lipoproteins cholesterol } \\
<0.9 \mathrm{mmol} / \mathrm{l}(>35 \mathrm{mg} / \mathrm{dL})\end{array}$ & 24.8 & 11.7 & $<0.001$ \\
\hline $\begin{array}{c}\text { Low density lipoproteins cholesterol } \\
>3.4 \mathrm{mmol} / \mathrm{l}(>130 \mathrm{mg} / \mathrm{dL})\end{array}$ & 72.8 & 84.4 & $<0.001$ \\
\hline
\end{tabular}

It should be especially noted hypertriglyceridemia and a decrease in the level of high-density lipoprotein cholesterol [911]. In patients with diabetes mellitus 2 , hyper production of "small, dense" low-density lipoproteins is isolated [12]. At the same time, $69 \%$ of patients with diabetes have lipid metabolism disorders [13]. The atherogenic effect of dyslipidemia is enhanced by the addition of diabetic disturbances in carbohydrate metabolism. For patients suffering from diabetes, the most typical lipid triad with diabetes: hypertriglyceridemia, an increase in the percentage of "small, dense" low-density lipoproteins, a decrease in high-density lipoprotein cholesterol. These changes in the lipid spectrum contribute to the development of atherosclerosis, regardless of the increase in the level of total cholesterol and the total fraction of low-density lipoproteins cholesterol.

Considering the important role of triglycerides in the development of dyslipidemia in diabetes mellitus, we cite the classification of serum triglycerides levels (Table 2) [14]. Great importance in the development of early coronary atherosclerosis has a specific violation of lipid metabolism in patients with diabetes 2 - diabetic dyslipidemia. 
Table 2: Classification of triglyceride levels in serum.

\begin{tabular}{|c|c|c|}
\hline Triglyceride levels & \multicolumn{2}{|c|}{ Value } \\
\hline & mmol/l & mg/dL \\
\hline Normal & $<1.7$ & $<150$ \\
\hline Borderline increased & $1.7-2.25$ & $150-199$ \\
\hline High & $2.3-5.6$ & $200-499$ \\
\hline Very high & $>5.6$ & $>500$ \\
\hline
\end{tabular}

Diabetes Mellitus, Ischemic Heart Disease and Hypertension

Epidemiological data suggest a reliable relationship between the level of glycosylated hemoglobin and the risk of cardiovascular morbidity and mortality. With an increase in the level of glycosylated hemoglobin by $1 \%$, the risk of cardiovascular morbidity increases by $10 \%$ [15].

Insulin resistance also plays a critical role in the pathogenesis of diabetes 2. Hyperinsulinemia is closely related to the metabolic syndrome, which includes insulin resistance, arterial hypertension and obesity and is accompanied by a high risk of coronary heart disease.

The change in the concentration of plasma lipids in diabetes mellitus 2 is a predictor of coronary heart disease. It was established that in persons with high blood glucose level in the blood on an empty stomach and after a load a significantly higher cardiovascular morbidity mortality was noted [16]. Asymptomatic hyperglycemia, especially in women, is an important risk factor for the development of coronary heart disease [17].

The effect of hyperinsulinemia and insulin resistance on the development of atherosclerosis is associated with the impact on blood coagulation processes [18]. Hypercoagulation and depression of fibrinolysis are noted, which can contribute to intracoronary thrombosis. In patients with diabetes mellitus 2 , damage to the endothelium and its dysfunction are detected, which is an additional factor in the increased risk of developing coronary artery disease.

The main cause of death of patients with diabetes is the coronary heart disease, up to $80 \%$. Mortality from myocardial infarction among patients with diabetes is 39\% [19], from stroke -exceeds that in people without diabetes [5]. With the combination of arterial hypertension and diabetes in 2-4 times the risk of developing coronary artery disease, which directly correlates with the duration of diabetes [20]. Arterial hypertension is found in $20-60 \%$ of patients with diabetes mellitus 2 , it occurs 1.5 times more often than in people without diabetes. The presence of arterial hypertension in diabetes increases the risk of macrovascular (coronary heart disease, cardiac failure, stroke) and microvascular (diabetic retinopathy, nephropathy) complications [21]. Important is the fact that in patients with $\mathrm{AH}$ and diabetes the benefits of antihypertensive therapy are more pronounced than in patients without diabetes. In this regard, strict control of blood pressure in this group of patients is extremely necessary.

With diabetes, the emergence of all forms of coronary heart disease, both painful, and, especially, painless. The presence of microangiopathies and neuropathies with diabetes promotes the formation of painless variants of coronary heart disease (painless myocardial infarction, atypical attacks of angina pectoris), proceeding in the form of disturbances of heart rhythm and heart failure. In patients with diabetes, 2-fold painless myocardial infarction is found, which is associated with autonomic cardiac neuropathy. With diabetes, the frequent form of coronary heart disease is painless myocardial ischemia. It consists in transient disorders of metabolism, function, perfusion, electrical activity of the myocardium, which are not manifested by attacks of angina pectoris or their equivalents.

Clinical features of coronary heart disease in patients with diabetes are as follows:

a) The same frequency in men and women;

b) High incidence of painless forms of coronary heart disease (painless myocardial ischemia, painless myocardial infarction);

c) Frequent development of postinfarction complications;

d) Mortality in acute (10 days) and subacute (4-8 weeks) periods myocardial infarction is 2 times higher than that in persons without diabetes.

In the presence of diabetes should strive for the possible elimination of all episodes of myocardial ischemia, and not only to arrest typical attacks of angina (getting rid of the "total ischemic burden" - total ischemic burden). This can be achieved by reducing the frequency and duration of episodes of ST-segment depression in Holter electrocardiogram monitoring, which should be used more widely in patients with diabetes to evaluate the effectiveness of anti-ischemic treatment.

\section{Diabetes Mellitus and Kidney Damage}

The earliest marker of kidney damage with diabetes is microalbuminuria, it is a harbinger of diabetic nephropathy and an important risk factor for the formation of cardiovascular pathology. Persistent albuminuria at the level of $30-299 \mathrm{mg} / 24 \mathrm{~h}$ (or microalbuminuria) serves as a marker of nephropathy and at the same time a marker of the risk of developing coronary heart disease [22].

\section{Diabetes Mellitus and Heart Failure}

The Framingham study quite convincingly confirmed the increased risk of developing heart failure in patients with diabetes - in men 4 times, in women - 8 times more often than in people without diabetes. According to the Russian EPOA study, the presence of $\mathrm{HF}$ is an important predictor of the development of diabetes in the future. In the general population, the prevalence 
of diabetes is $2.9 \%$, and among patients with heart failure III-IV pharmakokinetics $15.8 \%$. With chronic heart failure, the presence of insulin-independent diabetes significantly worsens the prognosis of patients, especially women (by 45\%).

With diabetes, the myocardium is affected regardless of the presence of coronary artery atherosclerosis (diabetic cardiomyopathy). Changes in the vessels of the heart are manifested in the form of microangiopathies, microcirculation is disturbed, morpho-functional changes in the myocardium are noted. The bioelectrical activity of the myocardium is disturbed, its contractility decreases with a tendency to the development of circulatory decompensation. With a prolonged course of diabetes, especially type 2 , atherosclerotic changes affect not only the main arteries, but also arteries of medium and small caliber. Also, in patients with diabetes 2 expressed myocardial hypertrophy, which contributes to the violation of the coronary circulation. Progression of left ventricular hypertrophy and dilatation reduces contractile function, wall tension occurs during systole, which increases myocardial oxygen demand and development of cardiosclerosis. With diabetes often develops a hyperkinetic variant of central hemodynamics with an increase in the volume of circulating blood, tachycardia [23].

Diabetic cardiomyopathy is manifested by hypertrophy of cardiomyocytes, myocardial fibrosis. The increased content of collagen in the myocardium, hypertrophy of the left ventricular leads to loss of elasticity with the appearance of rigidity, rigidity of the heart muscle.

Thus, the reduction in the contractility of the myocardium in diabetes and coronary artery disease is due to cardiac and noncardiac factors. Diabetic myocardial dystrophy is fundamentally associated with a disturbance in the metabolism of cardiomyocytes, and diabetic vegetoneuropathy and microangiopathy are also important [24]. Cardiac disorders in patients with diabetes mellitus 2 are also characterized by a diastolic left ventricular dysfunction. A number of pathogenetic mechanisms take part in the formation of the heart lesion in diabetes: macroangiopathy with coronary artery atherosclerosis, a violation of myocardial metabolism, microcirculatory disorders in the form of diabetic microangiopathy. The defeat of the heart with diabetes requires a preventive and curative effect.

\section{Treatment of Dyslipidemia in Patients with Diabetes Mellitus}

Correction of dyslipidemia with diabetes is carried out on the same principles as in patients with coronary heart disease. Statins are the first choice drugs. In this case, do not forget that improving the prognosis in patients with diabetes, ischemic heart disease and heart failure requires mandatory strict metabolic control of target levels of glucose and blood pressure. Control of glycemia is an important basis for the management of patients with diabetes. Clinical studies of DCCT [25], UKPDS [26] have shown that the improvement in glycemic control is accompanied by a steady decrease in the risk of retinopathy, nephropathy, and a significant reduction in the risk of microvascular complications by $25 \%$ and an unreliable trend towards a reduction in myocardial infarction risk by $16 \%$.

The goal of lipid-lowering therapy for diabetes is to achieve total cholesterol level $<4.5 \mathrm{mmol} / \mathrm{l}$, low-density lipoproteins cholesterol $<2.6 \mathrm{mmol} / \mathrm{l}$. The optimal level of high-density lipoprotein cholesterol is $1.02 \mathrm{mmol} / \mathrm{l}$ in men and $>1.28 \mathrm{mmol} / \mathrm{l}$ in women [27], the concentration of triglycerides is $<1.7 \mathrm{mmol} / \mathrm{l}$. In terms of lowering the level of low-density lipoproteins cholesterol, the leading role belongs to statins; Fibrates (fenofibrate, gemfibrazil, ciprofibrate, etc.) are particularly effective in decreasing the level of triglycerides and in increasing the level of high-density lipoprotein cholesterol. Patients with mixed hyperlipidemia are indicated the purpose of combined therapy (statins + fibrates).

Several meta-analyzes of recent years have established a diabetogenic effect of statin therapy [28-30], which is dosedependent and directly related to the presence of risk factors for cardiovascular morbidity. The question of prescribing statins to patients with diabetes has many nuances, requiring an individual approach. There are no doubts about the positive effects of prescribing statins. In patients with diabetes, preference should be given to prescribing statins with the most pronounced hypolipidemic effect -rosuvastatin and atorvastatin. The largest meta-analysis to assess the effect of lowering low-density lipoproteins cholesterol on the risk of cardiovascular events was performed in 2010 and included 26 clinical trials involving 170,000 patients [31]. The average follow-up time was 5.1 years. The analysis showed that a decrease in the low-density lipoproteins cholesterol level of $1 \mathrm{mmol} / \mathrm{l}$ leads to a $10 \%$ reduction in the risk of total death, a $20 \%$ reduction in the risk of cardiovascular mortality, an $11 \%$ reduction in the risk of cardiovascular events. In 2015, the recommendations of the American Association for the Treatment of diabetes (ADA), the main provisions of which largely repeat the provisions set out in the IAS and ACC / AHA. When prescribing statin therapy, the presence of a cardiovascular morbidity risk level is taken into account [32].

\section{Treatment of Arterial Hypertension in Patients with Diabetes Mellitus}

Most often, arterial hypertension and cardiovascular morbidity develop against the background of already existing diabetes mellitus 2. This combination carries the risk of rapid development and progression of coronary heart disease, characteristic for both arterial hypertension and diabetes, because the target organs for arterial hypertension and diabetes mellitus are the same: myocardium, coronary arteries, vessels of the brain, kidneys and retina. With such a combination, the incidence of coronary heart disease increases 3.5-4 times, acute cerebrovascular accident 3 times, chronic renal failure 30 times, complete loss of vision due to retinopathy 4-5 times compared with the isolated effect of arterial hypertension. 
The development of hypertension in patients with diabetes mellitus is associated with the activation of mechanisms regulating blood pressure, which primarily concerns the hyper activation of the sympathetic nervous system. Under the influence of sympathetic nervous system activation, the exchange of noradrenaline is disturbed, which leads to severe tissue hypersympathicotonia, sympathetic stimulation of the heart, blood vessels, kidneys, promoting blood pressure. Control of blood pressure, according to the International Federation of Diabetes (1999), reduces the risk of coronary heart disease in patients with diabetes by $51 \%$.

The target level of blood pressure in patients with diabetes is lower than in patients without diabetes - $130 / 80 \mathrm{~mm} \mathrm{Hg}$, and in the presence of severe nephropathy $-125 / 75 \mathrm{~mm} \mathrm{Hg}$. At achievement of such level it is possible to break progression of vascular complications at diabetes mellitus. The first choice drugs are angiotensin-converting enzyme (ACE inhibitors) and angiotensin II receptor antagonists (ARAII). Slow, prolonged calcium antagonists (amlodipine, normodipine, lacidipine, etc.) and cardioselective $\beta$-adrenoblockers (metoprolol, carvedilol, nebivolol, bisoprolol, etc.) can also be used.

In patients with diabetes with increased activity of SNS and arterial hypertension, it is advisable to use drugs that reduce its activation, in particular, $\beta$-adrenoblockers, which are widely used in diabetes 2 , especially when combined with arterial hypertension. Consequently, $\beta$-adrenoblockers, as well as ACE inhibitors, ARAII, prevent the development of cardiac complications in diabetes mellitus 2 . A number of $\beta$-blockers that have properties of selective $\beta$-adrenoblockers with vasodilating and protective activity are indicated for use in patients with diabetes mellitus and arterial hypertension. These drugs include: bisoprolol, nebivolol, carvedilol, etc. It is also advisable to use the combination of ARAII and calcium antagonist amlodipine in patients with diabetes mellitus 2 and arterial hypertension.

The importance of accounting for long-term antihypertensive therapy of metabolic effects, and in particular the development of diabetes, is emphasized in the latest European recommendations on arterial hypertension.

\section{Treatment of Chronic Heart Failure in Patients with Diabetes Mellitus}

Pathogenesis and treatment of chronic heart failure in patients with diabetes have certain characteristics that should be considered when conducting rational therapy. Treatment of heart failure in patients with diabetes generally corresponds to the generally accepted principles of therapy of chronic heart failure. However, an indispensable feature of the treatment of such patients is careful dynamic control over the basic metabolic parameters.

The main means of treatment of heart failure in diabetes are angiotensin-converting enzyme (ACE inhibitors) and angiotensin II receptor antagonists (ARAII), the efficacy of which is superior, according to the Russian study by FASON [33-35], therapy for decompensation of blood circulation in patients without diabetes.

In a meta-analysis (SARRR study), comparing the efficacy of angiotensin-converting enzyme (ACE inhibitors), $\beta$-blockers, AC and diuretics in the treatment of patients with arterial hypertension and diabetes mellitus, it was shown that the administration of angiotensin-converting enzyme (ACE inhibitors) and angiotensin II receptor antagonists (ARAII) significantly reduced the risk of acute myocardial infarction by $48 \%$, cardiovascular accidents by $32 \%$.

Priority is the modern cardio selective and non-selective $\beta$-blockers with the properties of indirect vasalators (metoprolol, nebivolol, bisoprolol, carvedilol, etc.). The hemodynamic effects of carvedilol are a reduction in total peripheral vascular resistance and preload, an increase in cardiac output without reflex tachycardia. In addition, the drug has antioxidant and antiarrhythmic activity, a vasoprotective effect. These properties make carvedilol very valuable in the treatment of patients with a combination of diabetes and chronic heart failure.

Active components reduce the frequency and duration of painless myocardial ischemia. It is preferable to use vasoselective and long-acting active components (amlodipine, felodipine, etc.) that do not cause reflex tachycardia, an increase in the level of catecholamines, and a pro -cemic effect. In addition, active components have an anti-atherogenic effect.

Among diuretics, potassium-sparing agents (aldactone, veroshpiron), indapamide are used; caution should be given to loop and thiazide diuretics - under the control of carbohydrate metabolism and the level of potassium in the blood. It is expedient to use metabolic means - trimetazidine, etc.

\section{References}

1. Shaw JE, Sicree RA, Zimmet PZ (2015) Global estimates of the prevalence of diabetes for 2010 and 2030. Diabetes Res Clin Pract 87(1): 4-14.

2. Fuller JH, Stevens LK, Wang SL (2011) Risk factors for cardiovascular mortality and morbidity: the WHO Mulinational Study of Vascular Disease in Diabetes. Diabctologia 44(Suppl 2): S54-S64.

3. Betteridge DJ (2012) Epidemiology of the cardiac complications of type 2 diabetes mellitus. Medicographia 23: 95-99.

4. Pyorala K (2005) Diabetes and coronary artery disease: what a coincidence? J Cardiovasc Pharmacol 16 (Suppl 9): S8-S14.

5. Buse JB, Ginsberg HN, Bakris GL, Clark NG, Costa F, et al. (2009) Primary prevention of cardiovascular diseases in people with diabetes mellitus: a scientific statement from the American Heart Association and the American Diabetes Association. Circulation 115(1): 114-126.

6. Wilson PW, Kannel WB (2015) Obesity, diabetes, and risk of cardiovascular disease in the elderly. Am J Geriatr Cardiol 11(2): 119125 .

7. Alexander CM, Antonello S (2012) Pract Diabet 21: 21-28.

8. Mykkanen L, Laakso M, Pyoral SK (2012) Asymptomatic hyperglycemia and atherosclerotic vascular disease in the elderly. Diabetes Care 15(8): 1020-1030. 
9. Laakso M (1995) Epidemiology of diabetic dyslipidemia. Diabetes Rev 3: 408-422.

10. Steiner G (2009) Risk factors for macrovascular disease in type 2 diabetes. Classic lipid abnormalities. Diabetes Care 22(Suppl 3): C6-C9.

11. Langsted A, Freiberg JJ, Nordestgaard BG (2013) Fasting and nonfasting lipid levels: influence of normal food intake on lipids, lipoproteins, apolipoproteins, and cardiovascular risk prediction. Circulation 118(20): 2047-2056.

12. Gerber PA, Thalhammer C, Schmied C, Spring S, Amann-Vesti B, et al. (2013) Small dense LDL particles predict changes in intitna media thickness and insulin resistance in men with type 2 diabetes and prediabetes - a prospective cohort study. PLoS One 8(8): e72763.

13. Slender M, Eaton S, Clark D, Hopkinson P (2013) Cardiovascular risk factors and outcomes in type 2 diabetes patients in primary care. The future of diabetes care. Selected Abstracts of the $36^{\text {th }}$ Annual Meeting from the Europian Association for the Study of Diabetes Poster.

14. (2012) Adult Treatment Panel III, ATP 111.

15. Barrett Connor EM (2015) Does hyperglycemia really cause coronary heart disease? Diabetes Care 20(10): 1620-1623.

16. Balkou B, Shipley M, Jarrett RJ, Pyörälä K, Pyörälä M, et al. (2014) High blood glucose concentration is a risk factor for mortality in middleaged nondiabetic men. Diabetes Care 21(3): 360-367.

17. Pyorala K, Pederson TR, Kjekshus J, Faergeman O, Olsson AG, et al. (1997) The Scandinavian Simvastatin Survival Study (4S) Group. Cholesterol lowering with simvastatin improves prognosis of diabetic patients with coronary heart disease. Diabetes Care 20(4): 614-620.

18. Sobel BE (2015) Coronary artery disease and fibrinolysis: from the blood to the vessel wall. Thromb Haemost 82(Suppl 1): 8-13.

19. Morrish NJ, Wang SL, Stevens LK, Fuller JH, Keen H (2013) Mortality and causes of death in the WHO multinational study of vascular disease in diabetes. Diabetologia 44(Suppl 2): S14-S21.

20. Lehto S, Ronnemaa T, Haffner SM, Pyörälä K, Kallio V, et al. (2012) Dyslipidemia and hyperglycemia predict coronary heart disease events in middle-aged patients with NIDDM. Diabetes 46(8): 1354-1359.

21. Sawicki PT, Heise T, Berger (2012) Antihypertensive treatment and mortality in diabetic patients. What is №e evidence? M Diabetologia 40(2): 34-37.

22. Parving HH (2013) Initiation and progression of diabetic nephropathy N Engl J Med 335(22): 1682-1683.

\section{Sokolov EI (2012) Diabeticheskoe serdce. M Medicina.}

24. Reaven GM, Lithell H, Landsberg L (2015) Hypertension and associated metabolic abnormalities-the role of insulin resistance and the sympathoadrenal system. N Eng J Med 334(6): 374-381.

25. The Diabetes Control and Complications THal (DCCT), National institute of diabetes and digestive and kidney diseases. 2002-2012.

26. (2010) UK prospective diabetes study group. Lancet 352: 837-853.

27. Lipton JA, Can A, Akoudad S, Simoons ML (2011) The role of insulin therapy and glucose normalisation in patients with acute coronary syndrome. Neth Heart J 19(2): 79-84.

28. Priess D, Seshasai SR, Welsh P, Murphy SA, Ho JE, et al. (2011) Risk of incident diabetes with intensive-dose compared with moderate-dose statin therapy: a meta-analysis. JAMA 305(24): 2556-2564.

29. Sattar N, Preiss D, Murray HM, Welsh P, Buckley BM, et al. (2010) Statin and risk of incident diabetes: a collaborative meta-analysis of randomized statin trials. Lancet 375(9716): 735-742.

30. Rajpathak SN, Kumbhani DJ, Crandall J, Barzilai N, Alderman M, et al. (2009) Statin therapy and developing type 2 diabetes: a meta-analysis. Diabetes Care 32(10): 2010-2014.

31. Boekholdt SM, Hovingh GK, Mora S, Arsenault BJ, Amarenco P, et al (2014) Very low levels of atherogenic lipoproteins and the risk for cardiovascular events: a meta-analysis of statin trials. J Am Coll Cardiol 64(5): 485-494.

32. American Diabetes Association (2015) Cardiovascular disease and risk management. Diabetes Care 38 (Suppl): 49-57.

33. (2011) FASON. Farmakojekonomicheskaja ocenka ispol'zovanija iAPF $\mathrm{v}$ ambulatornom lechenii bol'nyh s serdechnoj nedostatochnost'ju.

34. Allemann Y, Fraile B, Lambert M, Barbier M, Ferber P, et al. (2008) Efficacy of the combination of amlodipine and valsartan in patients with hypertension uncontrolled with previous monotherapy: the Exforge in Failure after Single Therapy (EX-FAST) study. J Clin Hypertens (Greenwich) 10(3): 185-194.

35. Wu HY, Huang JW, Lin HJ, Liao WC, Peng YS, et al. (2013) Comparative effectiveness of renin-angiotensin system blockers and other antihypertensive drugs in patients with diabetes: systematic review and bayesian network meta-analysis. BMJ 347: F6008.

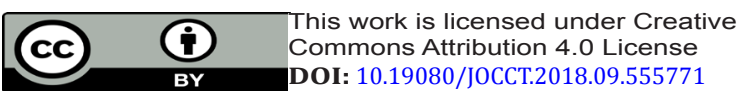

